\title{
A Multiplex-Multicast Scheme that Improves System Capacity of Voice- over-IP on Wireless LAN by $100 \%$ *
}

\author{
Wei Wang, Soung C. Liew, Qixiang Pang \\ Department of Information Engineering \\ The Chinese University of Hong Kong \\ \{wwang2, soung, qxpang \}@ie.cuhk.edu.hk \\ Victor O. K. Li \\ Department of Electrical and Electronic Engineering \\ The University of Hong Kong \\ vli@eee.hku.hk
}

\begin{abstract}
Voice-over-IP (VoIP) is an important application on the Internet. With the emergence of WLAN technology and its various advantages compared with the traditional wired LAN, it is fast becoming the "last-mile" of choice for the overall Internet infrastructure. This paper considers the support of VoIP over 802.11b WLAN. We show that although the raw WLAN capacity can potentially support more than 500 VoIP sessions, various overheads bring this down to only 12 VoIP sessions when using GSM 6.10 codec. We propose a novel multiplexing scheme for VoIP which exploits multicasting over WLAN for the downlink VoIP traffic. This scheme can achieve nearly $100 \%$ improvement in system capacity for both CBR and VBR voice sources, and for 802.11b, 802.11a, and 802.11g. In addition, we present results showing that the delay and delay jitter introduced by the proposed scheme are small. We believe that the scheme can reduce the blocking probability of VoIP sessions in an enterprise WLAN significantly.
\end{abstract}

Keywords - VoIP; Wireless LAN; 802.11; Access Point; Capacity; Multicasting; Multiplexing; Internet Telephone; Wireless Communications

\section{Introduction}

Voice over IP (VoIP) is one of the fastest growing Internet applications today [1]. It has two fundamental benefits compared with voice over traditional telephone networks. First, by exploiting advanced voice compression techniques and bandwidth sharing in packet-switched networks, VoIP can dramatically improve bandwidth efficiency. Second, it facilitates the creation of new services that combine voice communication with other media and data applications like video, white boarding and file sharing.

\footnotetext{
* This work is sponsored by the Areas of Excellence scheme established under the University Grant Committee of the Hong Kong Special Administrative Region, China (Project Number AoE/E01/99).
}

At the same time, driven by huge demands for portable access, the wireless LAN (WLAN) market is taking off quickly. Due to its convenience, mobility, and high-speed access, WLAN represents an important future trend for "lastmile" Internet access.

The most popular WLAN standard currently is IEEE $802.11 \mathrm{~b}$, which can support data rates up to $11 \mathrm{Mbps}$. A VoIP stream typically requires less than 10Kbps. Ideally, the number of simultaneous VoIP streams that can be supported by an $802.11 \mathrm{~b}$ WLAN is around $11 \mathrm{M} / 10 \mathrm{~K}=1100$, which corresponds to about 550 VoIP sessions, each with two VoIP streams. However, it turns out that the current WLAN can only support no more than a few VoIP sessions. For example, if GSM 6.10 codec is used, the maximum number of VoIP sessions that can be supported is 12 , a far cry from the estimate. This result is mainly due to the added packet-header overheads as the short VoIP packets traverse the various layers of the standard protocol stack, as well as the inefficiency inherent in the WLAN MAC protocol, as explained below.

A typical VoIP packet at the IP layer consists of 40-byte IP/UDP/RTP headers and a payload ranging from 10 to 30 bytes, depending on the codec used. So the efficiency at the IP layer for VoIP is already less than $50 \%$. At the 802.11 $\mathrm{MAC} / \mathrm{PHY}$ layers, the drop of efficiency is much worse. Consider a VoIP packet with 30-byte payload. The transmission time for it at $11 \mathrm{Mbps}$ is $30 * 8 / 11=22 \mu \mathrm{sec}$. The transmission time for the 40-byte IP/UDP/RTP header is $40 * 8 / 11=29 \mu \mathrm{sec}$. However, the $802.11 \mathrm{MAC} / \mathrm{PHY}$ layers have additional overhead of more than $800 \mu \mathrm{sec}$, attributed to the physical preamble, MAC header, MAC backoff time, MAC acknowledgement, and inter-transmission times of packets and acknowledgements. As a result, the overall efficiency drops to less than $3 \%$.

This paper proposes a voice multiplexing scheme to overcome the large overhead effect of VoIP in WLAN. Our scheme makes use of the features of the multicast mode of WLAN. We will show that the number of VoIP sessions that can be supported can be doubled with this simple technique, while maintaining small delay. 


\section{Background}

\subsection{VoIP Attributes}

For VoIP, the analog or PCM voice signals are encoded and compressed into a low-rate packet stream by codecs. Table 1 lists the attributes of several commonly used codecs. Generally, the codecs generate constant bit-rate audio frames consisting of 40-byte IP/UDP/RTP headers followed by a relatively small payload. We focus on the GSM 6.10 codec in this paper, although the general principle we propose is applicable to other codecs as well. For GSM 6.10, the payload is 33 bytes. The time between two adjacent frames is $20 \mathrm{~ms}$, corresponding to a rate of 50 packets per second per VoIP stream.

Table 1. Attributes of Commonly Used Codecs

\begin{tabular}{|c|c|c|c|c|c|}
\hline Codec & $\begin{array}{c}\text { GSM } \\
6.10\end{array}$ & G.711 & G.723.1 & G.726-32 & G.729 \\
\hline $\begin{array}{c}\text { Bit rate } \\
\text { (Kbps) }\end{array}$ & 13.2 & 64 & $5.3 / 6.3$ & 32 & 8 \\
\hline $\begin{array}{c}\text { Framing } \\
\text { interval (ms) }\end{array}$ & 20 & 20 & 30 & 20 & 10 \\
\hline $\begin{array}{c}\text { Payload } \\
\text { (Bytes) }\end{array}$ & 33 & 160 & $20 / 24$ & 80 & 10 \\
\hline Packets /sec & 50 & 50 & 33 & 50 & $50^{*}$ \\
\hline
\end{tabular}

* For all codecs except G.729, Packets/sec $=1 /$ (Framing interval). For G.729, two frames are combined into one packet so that Packets $/ \mathrm{sec}=1 /(2 *$ Framing interval $)$

\subsection{IEEE 802.11}

There are two access mechanisms specified in the IEEE 802.11 standard: Distributed Coordination Function (DCF) and Point Coordination Function (PCF). PCF is a centralized mechanism, where one central coordinator polls other stations and allows them contention free access to the channel. However, PCF is an option not supported in most commercial products.

DCF is based on the Carrier Sense Multiple Access with Collision Avoidance (CSMA/CA) protocol. The basic operation of 802.11 DCF is described in Fig. 1. Before transmission, a station will randomly choose a backoff time with number of time slots ranging from 0 to Contention Window (CW) -1. The station will decrease the backoff-timer counter progressively while the channel is idle after a DCF Inter Frame Space (DIFS) and pause the timer if it senses the channel to be busy. When the backoff value reaches zero, the station will transmit its packet.

If this is a unicast packet, the station will wait for the receiver to send back an ACK frame after a Short Inter Frame Space (SIFS) interval. If it does not receive the ACK, the station assumes the packet has been lost due to transmission errors or a collision. Thereafter, it doubles the $\mathrm{CW}$ value, generates a backoff time chosen randomly from the interval [0, CW-1], and retransmits this packet following the same procedure as above.
For a multicast or broadcast packet, the transmitting station will not wait for the ACK, as multicast receivers do not send back ACKs in general. There are no retransmissions for multicast and broadcast packets in 802.11 DCF. The station will proceed to send the next packet regardless of whether the earlier packet has been received successfully.

The values of the parameters of $802.11 \mathrm{~b}$ DCF are listed in Table 2 .
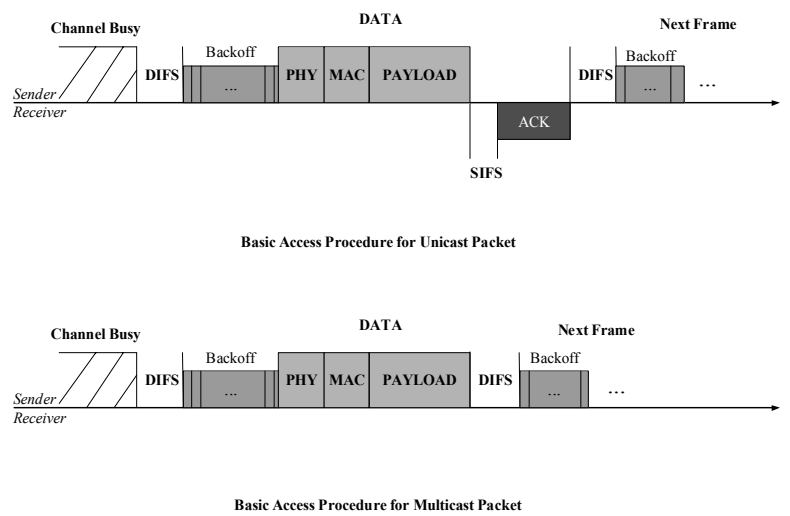

Figure 1. Basic Operation of 802.11 DCF

Although the maximum radio rate for $802.11 \mathrm{~b}$ is $11 \mathrm{Mbps}$, we found that some commercial products (e.g., Lucent Orinoco, Cisco) transmit multicast packet at $2 \mathrm{Mbps}$ bit-rate by default. This is due to the nature that in multicasting, the transmitter does not know who the receivers are. For backward compatibility, the sender uses 2 Mbps to transmit multicast packets so that the earlier versions of 802.11 products whose maximum data rate is 2 Mbps can receive them. There is usually a flag in the products to control this backward compatibility. We can simply disable this flag to use 11 Mbps multicast.

Table 2. Parameter Values of 802.11b DCF

\begin{tabular}{ll}
\hline DIFS & $50 \mu \mathrm{sec}$ \\
SIFS & $10 \mu \mathrm{sec}$ \\
Slot Time & $20 \mu \mathrm{sec}$ \\
CWmin & 32 \\
CWmax & 1023 \\
Data Rate & $1,2,5.5,11 \mathrm{Mbps}$ \\
Basic Rate & $2 \mathrm{Mbps}$ \\
PHY header* & $192 \mu \mathrm{sec}$ \\
MAC header & $34 \mathrm{bytes}$ \\
ACK* & $248 \mu \mathrm{sec}$
\end{tabular}

* PHY header is transmitted at $1 \mathrm{Mbps}$, ACK shown above is actually ACK frame + PHY header. The ACK frame is 14 bytes and is transmitted at basic rate, $2 \mathrm{Mbps}$, regardless of the data rate. 


\subsection{Related Work}

Previous work on VoIP over WLAN can be classified according to which access mechanism, DCF or PCF, is used. References [3] and [4] assumed the use of PCF. However, as mentioned above, PCF is not supported in most 802.11 products, and its popularity pales in comparison to DCF. A reason could be that the market does not see a compelling need for PCF. In addition, DCF is a technology that has been well tested and proven to be robust in the field. For example, when there are two overlapping WLANs using the same frequency channel, DCF will continue to work while PCF will not, since collisions between stations of the two WLANs may occur during their supposedly contention-free periods.

References [5 - 9] studied the use of DCF to support VoIP. Specifically, results in [6] and [7] confirm the existence of similar capacity limits as identified in this paper. However, no solutions are provided to improve the VoIP capacity over WLAN. References [5], [8] and [9] investigated various schemes for improving the VoIP capacity, but all the proposed schemes require modifications of the MAC protocol used by the VoIP stations. In contrast, our M-M scheme requires no changes to 802.11 MAC layers of the wireless end stations

There have been many schemes proposed for reliable multicast in general [10 - 12]. Most of them attempt to achieve $100 \%$ reliability by using some sort of retransmission strategies, at the expense of delay. Such approaches are not scalable and may cause VoIP to have unacceptable delay. Zero packet loss rate is too stringent a requirement for VoIP and is not necessary. Our paper demonstrates a simple scheme that solves the main cause for multicast packets losses in WLAN, namely, packet collisions. Specifically, in scheduling the transmission of multicast packets, our scheme 1) replaces DIFS with a Multicast Inter Frame Space (MIFS), with SIFS < MIFS < DIFS; 2) set the contention window, CW, to 1 . This solution can in principle be incorporated into mechanisms provided by the newly proposed 802.11 e standard.

\section{VoIP Multiplex-Multicast Scheme}

\subsection{System Architecture}

An 802.11 WLAN is referred to as the basic service set (BSS) in the standard specification. There are two types of BSSs: Independent BSS and Infrastructure BSS. Stations in an independent BSS communicate directly with each other. In contrast, stations in an infrastructure BSS communicate with each other via an Access Point (AP). That is, all traffic to and from a station must flow through the $\mathrm{AP}$, which acts as a base station.

This paper focuses on infrastructure BSSs. We assume that all voice streams are between stations in different BSSs, since users seldom call their neighbors in the same BSS. All voice traffic generated within a BSS is delivered to their called parties located at another BSS.

For illustration, let us consider the network architecture as shown in Fig. 2a. Each AP has two interfaces, an 802.11 interface which is used to communicate with wireless stations, and an Ethernet interface which is connected to the voice gateway. Two gateways for different BSSs are connected through the Internet. The voice gateway is required by the H.323 standard and is used for address translation, call routing for signaling and admission control purposes [1]. All voice packets will go through the gateway before entering the WLAN.

In the subsequent discussion, we will assume that our proposed voice multiplexer resides in the voice gateway. This is purely for the sake of having a concrete reference design for us to expound on the multiplex-multicast concept. In general, the functionality of the voice multiplexer could reside in the voice gateway, a specially-designed AP, or a server between the voice gateway and a general-purpose AP.

Within a BSS, there are two streams for each VoIP session. The uplink stream is for voice originating from the station to the AP. The downlink stream is for voice originating from the other side of the VoIP session to the station, which flows from the remote gateway to the local gateway, and then through the AP to the station.

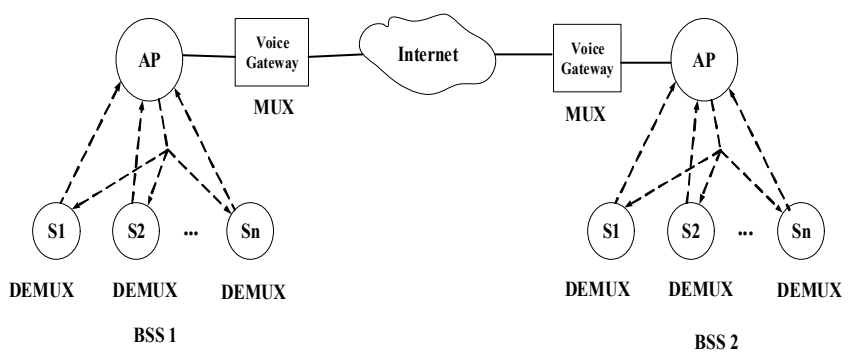

Figure 2a. Traffic Flows in Ordinary VoIP Scheme

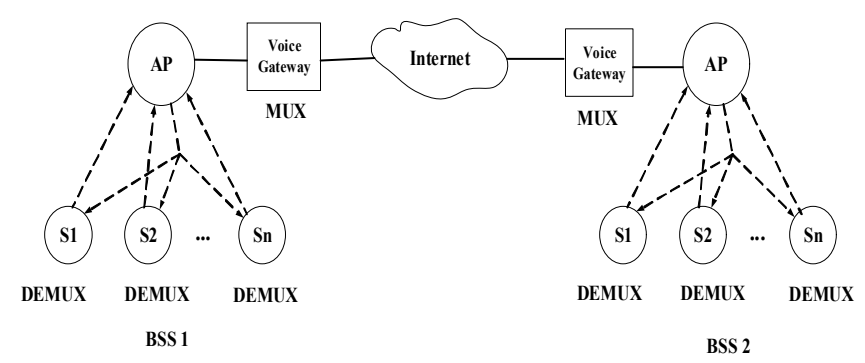

Figure 2b. Traffic Flows in VoIP Multiplex-Multicast Scheme 


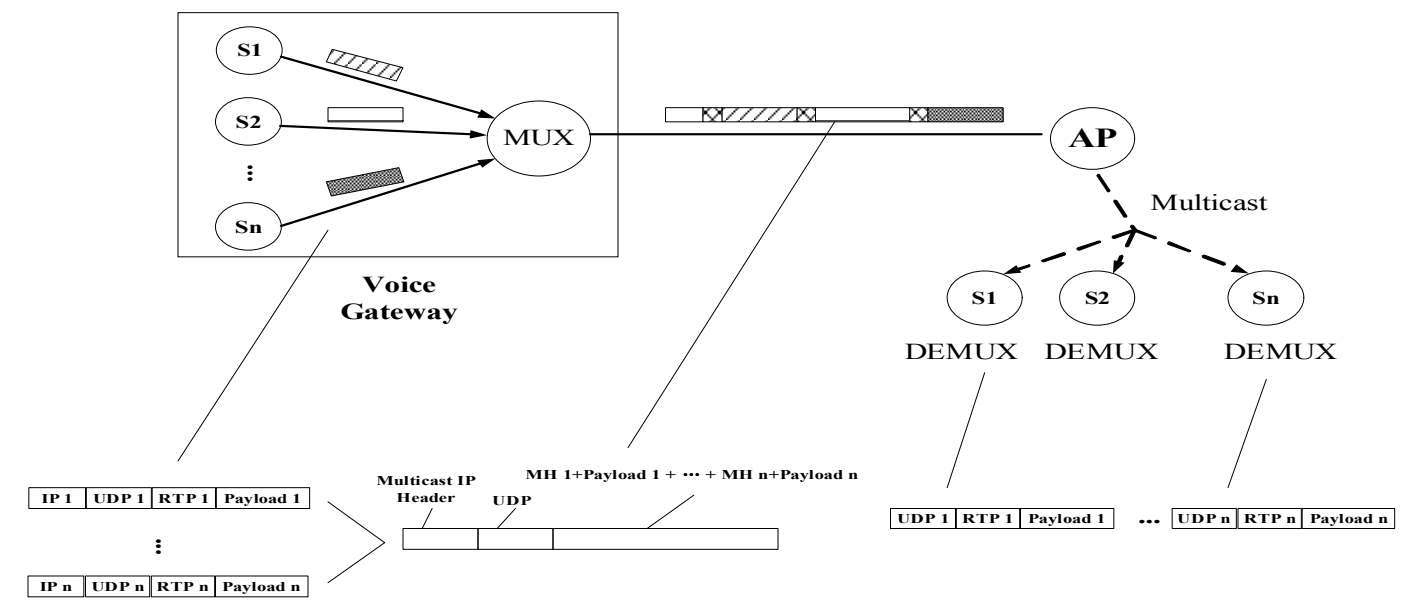

Figure 3. MUX/DEMUX Procedure

\subsection{Packet Multiplexing and Multicasting}

The main idea of our packet multiplex-multicast (M-M) scheme is to combine the data from several downlink streams into a single packet for multicast over the WLAN to their destinations. In this way, the overheads of multiple VoIP packets can be reduced to the overhead of one multicast packet.

The MUX and DEMUX procedures are illustrated in Fig. 3. Specifically, the downlink VoIP traffic first goes through a multiplexer (MUX) in the voice gateway. The MUX replaces the RTP, UDP and IP header of each voice packet with a compressed miniheader, combines multiple packets into a single multiplexed packet, then multicasts the multiplexed packet to the WLAN through the AP using a multicast IP address. All VoIP stations are set to be able to receive the packets on this multicast channel.

The payload of each VoIP packet is preceded by a miniheader in which there is an ID used to identify the session of the VoIP packet. The receiver for which the VoIP packet is targeted makes use of this ID to extract the VoIP packet out of the multiplexed packet. The extraction is performed by a demultiplexer (DEMUX) at the receiver. After retrieving the VoIP payload, the DEMUX then restores the original RTP header and necessary destination information, and assembles the data into its original form before forwarding it to the VoIP application. Other details of context mapping can be found in [13].

All the stations will use the normal unicasting to transmit uplink streams. The AP delivers the upstream packets it receives to the other BSS, whereupon the voice gateway at the other BSS sends the packets to their destinations using the same multiplexing scheme described above. From Fig. 2b, we see that this scheme can reduce the number of VoIP streams in one BSS from $2 n$ to $n+1$, where $n$ is the number of VoIP sessions.

The MUX sends out a multiplexed packet every $T$ ms, which is equal to or shorter than the VoIP inter-packet interval. For GSM 6.10, the inter-packet interval is $20 \mathrm{~ms}$.
Larger values of $T$ can improve bandwidth efficiency since more packets can be multiplexed, but the delay incurred will also be larger. For example, if $T=10 \mathrm{~ms}$, every two multiplexed packet contains one voice packet from each VoIP stream. The maximum multiplexing time for one voice packet is $10 \mathrm{~ms}$. If $T=20 \mathrm{~ms}$, every multiplexed packet contains one voice packet from each VoIP stream, and the maximum multiplexing time is $20 \mathrm{~ms}$. By adjusting $T$, one can control the tradeoff between bandwidth efficiency and delay.

Two aspects of VoIP multicasting over WLAN need to be addressed before we conclude this section. The first is the security implication. Since the multicast packets are received by all VoIP stations, a station could then extract VoIP packets not targeted for it and eavesdrop on others' conversations. However, VoIP multicasting over WLAN is no more insecure than regular unicast VoIP over WLAN. One could easily use a sniffer to collect all packets, unicast or multicast, in the WLAN - in fact, there are many free sharewares for doing that. The security problem in both cases should be solved by encrypting the voice packets.

The second aspect is that we have assumed in the above description that there is no additional delay other the MUX delay in the M-M scheme. It should be pointed out that when the power saving mode of 802.11 is turned on at some wireless stations, according to the 802.11 standard, multicast packets for them will be sent out at most only once every beacon period, after DTIM. Waiting for the next beacon will add additional delays to multicast packets. We do not advocate turning on of power saving mode for VoIP stations for this reason. Furthermore, power saving mode is effective only if traffic for the stations arrive at the AP sporadically, which is not the case with VoIP traffic. We have verified through experiments that for commercial products, if the power saving mode is not turned on, multicast packets are sent when they become available, and not after DTIM.

\subsection{Header Compression}

Besides aggregating VoIP streams, we can also increase the bandwidth efficiency by compressing the packet headers 
during multiplexing. The idea of RTP/UDP/IP header compression comes from two properties in most types of RTP streams. The first is that most of the fields in the IP, UDP and RTP headers do not change over the lifetime of an RTP session. Second, RTP header fields like sequence number and timestamp are increased by a constant amount for successive packets in a stream. So differential coding can be applied to compress these fields into fewer bits.

Our compression is similar to the scheme proposed in [13]. It depends on the use of context-mapping tables in MUX and DEMUX to record necessary information such as RTP header for future reconstruction, source IP address for differentiation between VoIP sessions, synchronization for proper (de)compression and (de)multiplexing. With this scheme, the RTP+UDP+IP header can be replaced with a 2-byte miniheader for most voice packets. We refer the reader to [13] for details. The major reason for the improved efficiency of our system here is the MUX/DEMUX scheme rather than the header compression scheme.

\section{Capacity Analysis}

In this section, we consider both continuous-bit-rate (CBR) and variable-bit-rate (VBR) voice sources. For CBR sources, voice packets are generated at the voice codec rate (e.g., 50 packets per second when GSM 6.10 is used). We model VBR sources using the Brady's ON-OFF model [19], in which data is generated at the voice codec rate during the ON state, and no data is generated during the OFF state. As in [19], we assume the ON and OFF times to be exponentially distributed with means of $1 \mathrm{sec}$ and $1.35 \mathrm{sec}$, respectively. We first consider the CBR case in the following capacity analysis.

\subsection{VoIP Capacity Analysis for 802.11b}

Let $n$ be the maximum number of sessions that can be supported. The transmission times for downlink and uplink packets are $T_{\text {down }}$ and $T_{u p}$, respectively. Let $T_{a v g}$ be the average time between the transmissions of two consecutive packets in a WLAN. That is, in one second, there are totally $1 / T_{\text {avg }}$ packets transmitted by the AP and all the stations. So,

$$
\begin{gathered}
1 / T_{\text {avg }}=\text { number of streams } * \text { number of packets sent by } \\
\text { one stream in one second. }
\end{gathered}
$$

\section{Capacity of Ordinary VoIP over WLAN}

For a VoIP packet, the header overhead $O H_{h d r}$ consists of the headers of RTP, UDP, IP and 802.11 MAC layer:

$$
O H_{h d r}=H_{R T P}+H_{U D P}+H_{I P}+H_{M A C}
$$

Besides, at the MAC layer, the overhead incurred at the sender is

$$
O H_{\text {sender }}=D I F S+\text { average } C W+P H Y
$$

If it is the unicast packet, the overhead incurred at the receiver is

$$
O H_{\text {receiver }}=S I F S+A C K
$$

where average $C W=\operatorname{slotTime} *\left(C W_{\min }-1\right) / 2$ is the average backoff time when there are no other contending stations. We ignore the possibility of collisions and the increase of backoff time in subsequent retransmissions after a collision in the analysis here. This means that the VoIP capacity we derive is an upper bound on the actual capacity. However, contention overhead is negligible compared with other overheads, and the analytical upper bound is actually a good approximation of the actual capacity, as will be verified by our simulation results later. So, we have

$$
\begin{aligned}
T_{\text {down }}=T_{u p}= & \left(\text { Payload }+\mathrm{OH}_{h d r}\right) * 8 / \text { dataRate } \\
& +O H_{\text {sender }}+O H_{\text {receiver }}
\end{aligned}
$$

In the ordinary VoIP case, we have $n$ downlink and $n$ uplink unicast streams. On average, for every downlink packet, there is a corresponding uplink packet. So,

$$
T_{\text {avg }}=\left(T_{\text {down }}+T_{\text {up }}\right) / 2
$$

From (1), we have

$$
1 / T_{\text {avg }}=2 n * N_{p}
$$

where $N_{p}$ is the number of packets sent by one stream per second.

The values of $D I F S, P H Y, S I F S, A C K$ for 802.11b are listed in Table 2. Assuming GSM 6.10 is used, Payload is 33 bytes, $N_{p}$ is 50. dataRate is 11 Mbps. Solving (7), we get $n=11.2$. We see that $802.11 \mathrm{~b}$ WLAN can only support around 11 VoIP sessions from the analysis.

\section{Capacity of Multiplex-Multicast Scheme over WLAN}

In this case, the RTP, UDP and IP header of each unmultiplexed packet is compressed to 2 bytes. $n$ packets are aggregated into one packet and they share the same header overhead, which includes UDP, IP and MAC headers of the multiplexed packet. There is no RTP header in the multiplexed packet. In addition, since the multiplexed packet is sent using multicast, it does not have $O H_{\text {receiver }}$. So,

$$
\begin{aligned}
T_{\text {down }}= & {\left[(\text { Payload }+2) * n+H_{U D P}+H_{I P}+H_{M A C}\right] } \\
& * 8 / \text { dataRate }+O H_{\text {sender }}
\end{aligned}
$$


Here on average, for one downlink packet, there are totally $n$ corresponding uplink packets. We have

$$
T_{\text {avg }}=\left(T_{\text {down }}+n * T_{u p}\right) /(n+1)
$$

where $T_{u p}$ is the same as (5). Solving (8) and (9) with

$$
1 / T_{\text {avg }}=(n+1) * N_{p},
$$

we get $n=21.2$.

We also derive the capacities when other codecs than GSM 6.10 are used in a similar way, and the results are listed in Table 3. We see that for most of the codecs, the M-M scheme can nearly double the capacity.

Table 3. VoIP Capacities assuming Different Codecs

\begin{tabular}{|c|c|c|}
\hline Codecs & Ordinary VoIP & $\begin{array}{c}\text { Multiplex-Multicast } \\
\text { Scheme }\end{array}$ \\
\hline GSM 6.10 & 11.2 & 21.2 \\
\hline G.711 & 10.2 & 17.7 \\
\hline G. 723.1 & 17.2 & 33.2 \\
\hline G. 726-32 & 10.8 & 19.8 \\
\hline G. 729 & 11.4 & 21.7 \\
\hline
\end{tabular}

Note that in the above, we assume the average $\mathrm{CW}$ wait time to be 15.5 time slots (i.e., $\left.\left(C W_{\min }-1\right) / 2\right)$. When there is more than one station, the average $\mathrm{CW}$ wait time is in fact smaller than this. This accounts for the observation in our simulations (see Table 6) that the maximum session is actually a little bit larger, even though we have ignored the possibility of increase in backoff time due to collisions in our analysis.

\subsection{VoIP Capacity Analysis for 802.11a and 802.11g}

$802.11 \mathrm{a}$ uses the same MAC protocol as $802.11 \mathrm{~b}$ but with a different set of parameters. In $802.11 \mathrm{a}$, the PHY preamble and the contention time slot are shorter, and the maximum data rate is much larger (see Table 4). Therefore, 802.11a may have a higher system capacity for VoIP. 802.11a, however, is not compatible with 802.11 b.

$802.11 \mathrm{~g}$ also has the same maximum data rate as $802.11 \mathrm{a}$. However, it has two different operation modes. In the $802.11 \mathrm{~g}$-only mode, all stations in the WLAN are $802.11 \mathrm{~g}$ stations, so that they can operate in a way that is more efficient but not compatible with 802.11b. In the 802.11bcompatible mode, some stations in the WLAN are $802.11 \mathrm{~b}$ stations, and $802.11 \mathrm{~g}$ stations must operate in a way that is compatible with $802.11 \mathrm{~b}$.

In the 802.11 g-only mode, timing spaces even smaller than those in 802.11a are used (Table 4), leading to a slightly higher capacity than 802.11 a. However, the use of $802.11 \mathrm{~g}$ only mode in practice is unlikely given the large installed base of $802.11 \mathrm{~b}$ equipment already in use. After all, the main motivation for the use of $802.11 \mathrm{~g}$ over $802.11 \mathrm{a}$ is that $802.11 \mathrm{~g}$ is compatible with $802.11 \mathrm{~b}$ while $802.11 \mathrm{a}$ is not. One would expect $802.11 \mathrm{~g}$ stations to mostly operate in the $802.11 \mathrm{~b}$ compatible mode in the field.

Although in the $802.11 \mathrm{~b}$-compatible mode of $802.11 \mathrm{~g}$, the maximum data rate of $54 \mathrm{Mbps}$ is much larger than the 11 Mbps of $802.11 \mathrm{~b}$, the other overheads are comparable. For packets with large payload, higher throughput than that in $802.11 \mathrm{~b}$ can be achieved. Unfortunately, VoIP packets have very small payload. As a result, the higher data rate of 54 Mbps does not yield much improvement as far as VoIP capacity is concerned, since the dominant overheads are not reduced. The following paragraph elaborates the operation of the $802.11 \mathrm{~b}$-compatible mode.

In the $802.11 \mathrm{~b}$-compatible mode, the DIFS, SIFS and contention slot time are the same as those in $802.11 \mathrm{~b}$, so that $802.11 \mathrm{~g}$ and $802.11 \mathrm{~b}$ stations can contend for the access of the channel in a fair manner. Furthermore, $802.11 \mathrm{~g}$ has to enable "protection", wherein the $802.11 \mathrm{~g}$ stations operating at the higher data rate must reserve the channel before accessing it at the higher speed using a slower reservation mechanism understandable by the $802.11 \mathrm{~b}$ stations.

There are two kinds of protections. The first is CTS-toself, in which an $802.11 \mathrm{~g}$ station needs to send a Clear-ToSend (CTS) frame to clear the channel before transmitting a data frame. This CTS frame is sent at the $802.11 \mathrm{~b}$ basic rate using the $802.11 \mathrm{~b}$ PHY preamble so that $802.11 \mathrm{~b}$ stations as well as other $802.11 \mathrm{~g}$ stations can hear it. The NAV value in the CTS frame specifies how long the channel will be reserved. The CTS-to-self mode is not targeted for solving the hidden node problem. For that, the RTS-CTS protection mode is used, in which the receiving station must return an RTS frame after the CTS frame before the transmitting station begins transmission.

Using the parameters listed in Table 4, we have performed the capacity analysis for $802.11 \mathrm{a}$ and $802.11 \mathrm{~g}$ based on essentially the same set of equations as in the previous section. The results for GSM 6.10 codec with CBR voice source are listed in Table 5.

As expected, 802.11g-only mode can achieve even higher capacity than 802.11a, thanks to its smaller DIFS and SIFS. However, when $802.11 \mathrm{~g}$ needs to be compatible with $802.11 \mathrm{~b}$, the capacity decreases drastically. In particular, when $802.11 \mathrm{~g}$ adopts RTS-CTS protection, the capacity is decreased to almost the same as in $802.11 \mathrm{~b}$. This shows that the higher data rate of $802.11 \mathrm{~g}$ fails to bring about a corresponding higher VoIP.

It is worthwhile to note that for all the variants of 802.11, the M-M scheme can achieve roughly the same percentage of improvement in VoIP capacity. That is, an improvement of slightly less than $100 \%$.

\subsection{VoIP Capacity with VBR Sources}

VBR encoding can reduce the traffic of VoIP streams so that the capacity for VBR VoIP will be larger in WLAN. For Brady's VBR model, the assumed mean ON time is 1 second, and the mean OFF time is 1.35 second. On average, the traffic 
load of VBR is $O N /(O N+O F F)=42.5 \%$ of the traffic load of CBR. The VBR VoIP capacity is simply

$$
C_{V B R}=C_{C B R} / \rho
$$

where $C_{C B R}$ is the capacity for CBR source, $\rho=O N /(O N+O F F)=42.5 \%$. The ordinary VBR VoIP capacity is $11.2 / 42.5 \%=26.3$, and the MultiplexMulticast VBR VoIP capacity is $21.2 / 42.5 \%=49.8$.

Table 4. Parameter Values of 802.11a and 802.11g

\begin{tabular}{|c|c|c|c|}
\hline & \multirow{2}{*}{$802.11 \mathrm{a}$} & \multicolumn{2}{|c|}{$802.11 \mathrm{~g}$} \\
\hline & & $\begin{array}{l}802.11 \mathrm{~g}- \\
\text { only }\end{array}$ & $\begin{array}{l}802.11 b- \\
\text { compatible }\end{array}$ \\
\hline DIFS & 34 us & 28 us & 50 us \\
\hline SIFS & 16 us & $10 \mathrm{us}$ & 10 us \\
\hline Slot Time & 9 us & 9 us & 20 us \\
\hline CWmin & 16 & 16 & 16 \\
\hline RTS & 14 bytes & 14 bytes & 14 bytes \\
\hline CTS & 14 bytes & 14 bytes & 14 bytes \\
\hline $\begin{array}{l}\text { Maximum } \\
\text { Data Rate }\end{array}$ & $54 \mathrm{Mbps}$ & $54 \mathrm{Mbps}$ & $54 \mathrm{Mbps}$ \\
\hline Basic Rate & $\mathrm{N} / \mathrm{A}$ & $\mathrm{N} / \mathrm{A}$ & $2 \mathrm{Mbps}$ \\
\hline $\begin{array}{l}\text { PHY for } \\
\text { protection } \\
\text { frames * }\end{array}$ & $\mathrm{N} / \mathrm{A}$ & $\mathrm{N} / \mathrm{A}$ & 192 us \\
\hline $\begin{array}{r}\text { PHY for } \\
\text { other frames }\end{array}$ & 20 us & 20 us & 20 us \\
\hline ACK frame & 24 us & 24 us & 24 us \\
\hline
\end{tabular}

* Protection frames are RTS, CTS frames used in $802.11 \mathrm{~b}-$ compatible mode of $802.11 \mathrm{~g}$

Table 5. VoIP Capacities for $802.11 \mathrm{~b}, 802.11 \mathrm{a}$ and 802.11g Derived from Analysis

\begin{tabular}{|c|c|c|}
\hline MAC & Ordinary VoIP & $\begin{array}{c}\text { Multiplex- } \\
\text { Multicast Scheme }\end{array}$ \\
\hline $802.11 \mathrm{~b}$ & 11.2 & 21.2 \\
\hline $802.11 \mathrm{a}$ & 56.4 & 108.8 \\
\hline 802.11 g-only & 60.5 & 116.5 \\
\hline $\begin{array}{c}\text { 802.11g with CTS- } \\
\text { to-self protection }\end{array}$ & 18.9 & 36.6 \\
\hline $\begin{array}{c}\text { 802.11g with RTS- } \\
\text { CTS protection }\end{array}$ & 12.7 & 24.3 \\
\hline
\end{tabular}

\subsection{Simulations}

We have validated our capacity analysis of $802.11 \mathrm{~b}$ by simulations. The simulator $\mathrm{ns}-2[20]$ is used. In the simulations, we only consider the local part (BSS1 plus the corresponding voice gateway) of the network shown in Fig. $2 \mathrm{a}$, since our focus is on WLAN, not the Internet. The payload size and frame generation interval are those of the GSM 6.10 codec.

We increase the number of VoIP sessions until the per stream packet loss rate exceeds $1 \%$. We define the system capacity to be the number of VoIP sessions that can be supported while maintaining the packet loss rate of every stream to be below $1 \%$. In our simulations, we assume that the retry limit for each packet is 3 . In other words, after a packet is retransmitted three times, it will be discarded regardless of whether the last transmission is successful. Commercial products by Orinoco, for example, adopt a retry limit of 3 .

For ordinary VoIP over WLAN, the simulations yield capacities of 12 and 25 for CBR and VBR, respectively. These results match the analysis very well. We also tried to increase the number of sessions by one beyond the capacity. We observed that this leads to a large surge in packet losses for the downlink streams. For example, for CBR, when the $13^{\text {th }}$ session is added, the packet loss rate for downlink streams abruptly jumps to around $6 \%$, while the loss rate for the uplink is still below $1 \%$.

This result is due to the symmetric treatment of all stations in 802.11: the AP is not treated differently from other stations as far as the MAC layer is concerned. For ordinary VoIP over WLAN, the AP needs to transmit $n$ times more traffic than each of the other stations. When $n$ is smaller than the system capacity, there is sufficient bandwidth to accommodate all transmissions of the AP. However, when $n$ exceeds the system capacity, since all stations including the AP are treated the same, the "extra" traffic from the AP will be curtailed, leading to a large packet loss rate for downlink VoIP streams.

This observation provides an alternative explanation as to why the M-M scheme can improve the VoIP capacity. With $n$ VoIP packets multiplexed into one packet, the AP traffic in terms of number of packets per second is reduced to the same as the traffic of each of the other stations.

The results of the M-M scheme are also listed in Table 6 . The simulation shows that the CBR capacity can be improved to 22, which matches analysis quite well. However, the VBR capacity can only be improved to 36 , which is far below the result of analysis. This can be explained as follows.

Recall that in the analysis we have ignored collisions. For CBR sources, the generated traffic is smooth and collision probability does not go up drastically as the number of VoIP sessions increase. In fact, the collision probability remains negligible right up to the capacity limit. However, for VBR sources, the traffic is bursty. Our analysis for VBR was based on the average traffic load. But the actual "instantaneous" traffic load fluctuates over time, depending on the number of ON sources. Even when the average traffic load is well below capacity, the instantaneous traffic load could reach a level 
beyond the throughput limit of WLAN to cause high collision probability.

Thanks to link-layer ARQ, unicast frame can tolerate several collisions before being discarded. The lack of ARQ in WLAN multicast, however, means that multicast frames will be dropped after the first collision. So when our M-M scheme is applied on VBR sources, the capacity is actually limited by the higher propensity for collision loss of downlink multicast frames. Fortunately, we can solve it by applying a minor modification on the AP MAC layer to reduce the collision probability of multicast frames. We refer to the solution as the MAC-layer Multicast Priority scheme (MMP). With MMP, when the AP has a multicast frame to transmit, instead of waiting for DIFS and then a contention backoff period, it just waits for a Multicast Inter-Frame Space (MIFS), before transmission. The contention backoff period is omitted altogether. The value of MIFS should be a value less than DIFS but larger than SIFS. By setting it larger than SIFS, it will not collide with control frames such as ACK and CTS. By making it smaller than DIFS and getting rid of the contention backoff period, collisions with unicast uplink packets are eliminated. In our simulation experiment, we set MIFS to be 30 us. Note that MMP is a general solution to the multicast collision problem in WLAN. That is, it is not limited to just VoIP multicasting. The restriction is that there should be no more than one multicast node within the WLAN; otherwise, multicast packets from different nodes would still collide. However, we believe in most multicast applications in an infrastructure-mode WLAN, the AP is likely to be the node from which multicast data is delivered to the clients. This modification allows the M-M VBR VoIP scheme to have capacity of 46, which is closer to the analytical result in Table 6.

Table 6. Analysis vs. Simulation: Capacity of Ordinary VoIP and Multiplex-Multicast Schemes assuming GSM 6.10 codec

\begin{tabular}{|c|r|r|r|c|}
\hline \multirow{2}{*}{$\begin{array}{c}\text { Different } \\
\text { Schemes }\end{array}$} & \multicolumn{2}{|c|}{ CBR } & \multicolumn{2}{c|}{ VBR } \\
\cline { 2 - 5 } & Analysis & Simulation & Analysis & Simulation \\
\hline Original VoIP & 11.2 & 12 & 26.3 & 25 \\
\hline $\begin{array}{c}\text { Multiplex- } \\
\text { Multicast } \\
\text { Scheme }\end{array}$ & 21.2 & 22 & 49.8 & $36^{*}$ \\
\hline
\end{tabular}

* After applying MMP, the capacity is actually 46 with loss and delay metric

\section{Delay Performance}

The previous section studied VoIP capacities over WLAN based on a packet-loss rate target of $1 \%$. To provide good voice quality, besides low packet-loss rates, we also need to consider the delay performance. In the following, we present results on the local delays incurred at the voice gateway and the WLAN.
With ordinary VoIP, the access delay within the WLAN is the only local delay. At the AP, the access delay of a VoIP packet is the time between its arrival to the AP until it is either successfully transmitted over the WLAN or dropped at the head of the queue because it has exhausted the retry limit for retransmissions. At the client, the access delay of a VoIP packet is time from when the packet is generated until it leaves the interface card, either due to successful transmission or exhaustion of the retry limit.

With the M-M scheme, in addition to the aforementioned access delay, the local delay for the downlink also includes the MUX delay incurred at the VoIP multiplexer. The MUX delay is the time from the arrival of a VoIP packet to the multiplexer until the time at which the next multiplexed packet is generated. With a multiplexing interval of $20 \mathrm{~ms}$, for example, the MUX delays are distributed between $0 \mathrm{~ms}$ and $20 \mathrm{~ms}$.

From an end-to-end viewpoint, it is essential for the local delay to be small so that the overall end-to-end delay of a VoIP stream can be bounded tightly to achieve good quality of service. As a reference benchmark for our delay investigations in this paper, we set a requirement that no more than $1 \%$ of the downlink or uplink VoIP packets should suffer a local delay of more than $30 \mathrm{~ms}$. This allows ample delay margin for delay in the backbone network for an end-to-end delay budget of 125 ms [2].

\subsection{Access Delay}

Figure 4a shows the access delays of successive packets of one randomly chosen CBR VoIP session in the ordinary VoIP scheme when there are 12 simultaneous CBR VoIP sessions (i.e., the system capacity is fully used). The graph on the left is the access delay incurred by the downlink traffic in the AP, while the graph on the right is the access delay incurred by the uplink traffic in its wireless station.

The average delay and delay jitter (defined to be the standard deviation of delay) in the AP are $2.5 \mathrm{~ms}$ and $1.4 \mathrm{~ms}$, respectively. The average delay and delay jitter in the wireless station are $1.2 \mathrm{~ms}$ and $1.0 \mathrm{~ms}$, respectively. The three-sigma delays (i.e., average delay $+3 *$ standard deviation) in the AP and wireless stations are therefore $6.7 \mathrm{~ms}$ and $4.2 \mathrm{~ms}$, respectively. This means that if the delays were to be normally distributed, less than $(1-99.73 \%)=0.27 \%$ of the packets would suffer local delays larger than $30 \mathrm{~ms}$. Thus, we see that even when the VoIP capacity is fully used, the local delay requirement can be met comfortably.

In addition to delay jitter, we can also look directly at the probability of access delay being smaller than a value. Table 7 tabulates such delay distributions, where $A$ is the random variable representing the access delay. Again, it shows that the requirement of less than $1 \%$ of packets having more than 30 ms delay can be met comfortably.

Figure $4 \mathrm{~b}$ shows the access delay when the M-M scheme is adopted, and the number of VoIP sessions is equal to the previously found capacity of 22 . The average delay and delay jitter for the AP (about $0.9 \mathrm{~ms}$ and $0.2 \mathrm{~ms}$ ) and the wireless 
stations (about $2.0 \mathrm{~ms}$ and $1.5 \mathrm{~ms}$ ) can still comfortably meet the three-sigma metric. From the left side of Fig. 4b, we can see the effect of multicasting downlink packets. Since there are no link layer retransmissions for the packets when collisions occur, the delays at the AP are quite smooth compared with the delays at the client (right side of Fig. 4b), where the uplink VoIP packets are transmitted using unicast. The probability of local delay being less than $30 \mathrm{~ms}$ will be presented later in Section 5.2, in which we add the multiplexing delay to the access delay to arrive at the actual local delay in the M-M scheme.
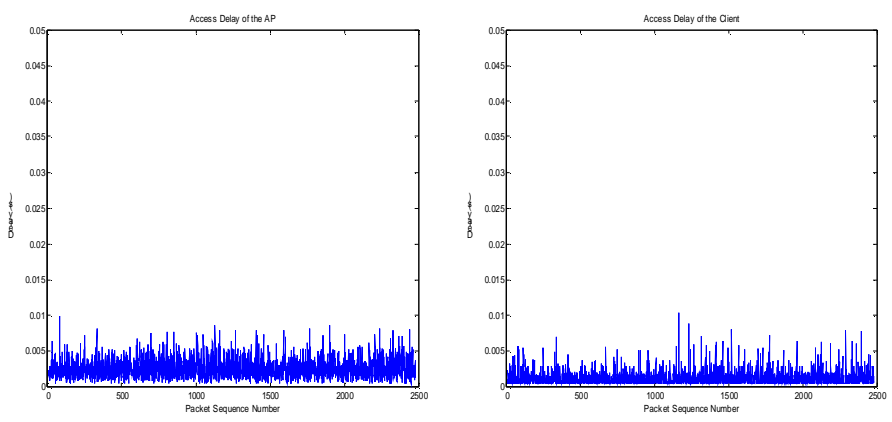

Figure 4a. Access Delays in AP and a Station in Original VoIP over WLAN when there are 12 Sessions
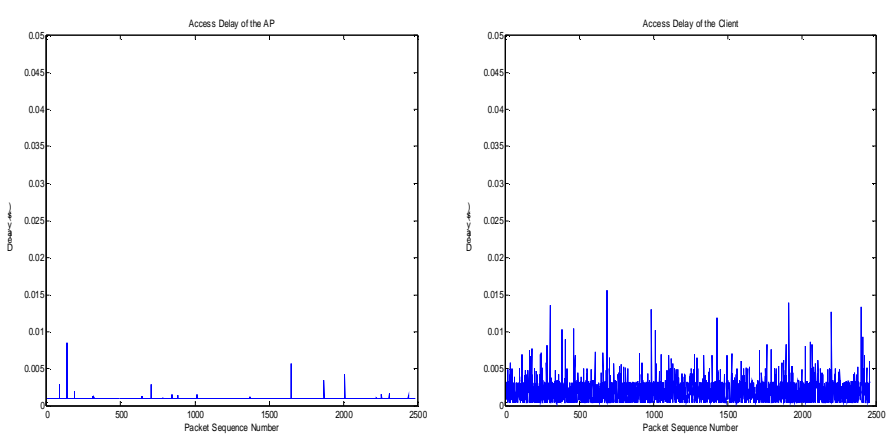

Figure 4b Access Delay in AP and a Station in M-M Scheme when there are 22 Sessions

Figure 4. Delays for CBR VoIP over WLAN

We now look at the performance when VBR encoding is used. Figure 5a shows the delay of ordinary VBR VoIP over WLAN. The average delay and jitter for AP (about $3.6 \mathrm{~ms}$ and $5.9 \mathrm{~ms}$ ) and those of the wireless station (about $1.4 \mathrm{~ms}$ and 1.3 ms) are still acceptable. However, even though the AP delay meets the three-sigma metric, we find that $1 \%$ of the downlink packets have delays larger than $30 \mathrm{~ms}$ (see Table 7). This is because the delay is not normally distributed due to the burstiness of the traffic.

Figure $5 \mathrm{~b}$ shows the delay of the M-M scheme for VBR VoIP when there are 36 sessions. The average delay and delay jitter for AP are 1.1 and $0.7 \mathrm{~ms}$, respectively, and those for the station are 0.9 and $0.7 \mathrm{~ms}$, respectively. The low values of the delay figures suggest that the channel is not fully utilized. Recall that the system capacity of 36 sessions was derived from our simulation results in which we required the packet loss rate to be less than $1 \%$. The results from Fig. $5 \mathrm{~b}$ show that the capacity is limited by that loss-rate requirement rather than the delay requirement, and in principle the capacity can be increased if a way can be found to lower the loss rate. Fig. 5c shows the delay performance when the capacity is increased to 46 after applying the MMP scheme we proposed in the previous section. Since the downlink multicast packet has priority over other unicast packet within the WLAN, the access delay of the AP is very small and smooth, as shown in the left part of Fig. 5c.
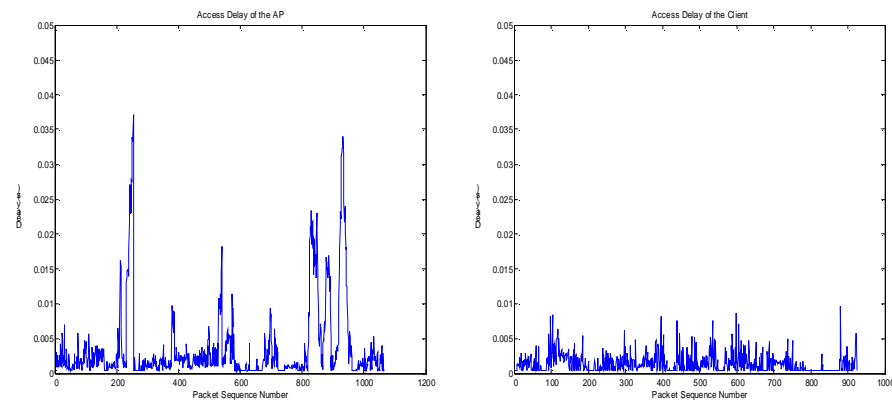

Figure 5a Access Delay in AP and a Station in Original VoIP over WLAN when there are 25 Sessions
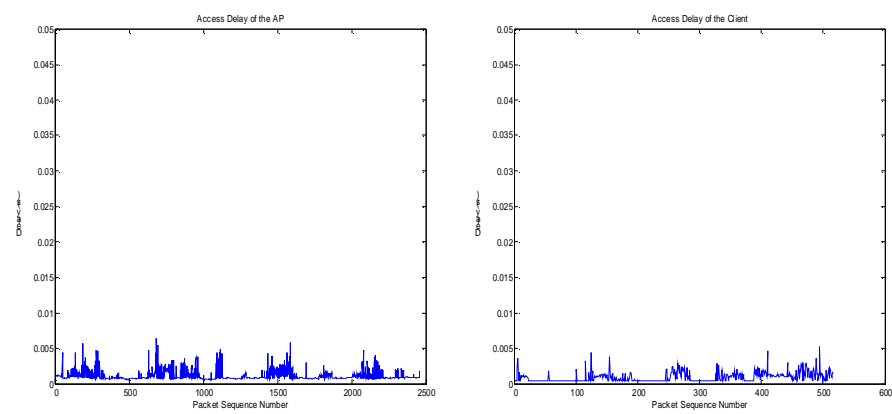

Figure 5b Access Delay in AP and a Station in M-M Scheme when there are 36 Sessions
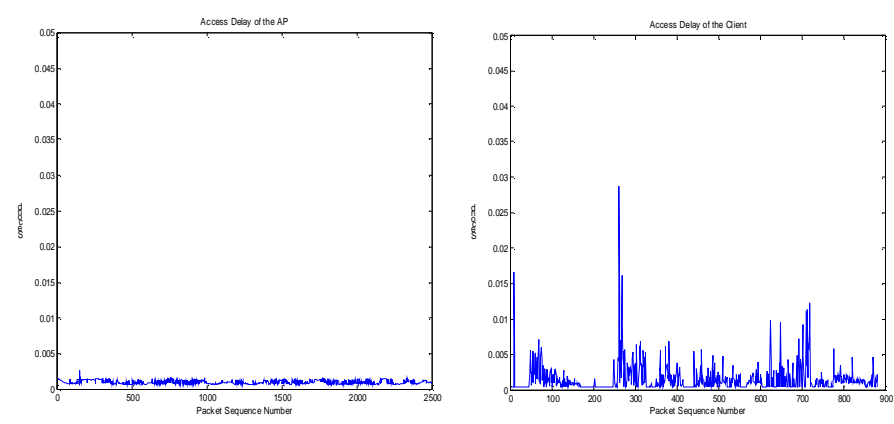

Figure 5c Access Delay in AP and a Station in M-M Scheme when there are 46 Sessions

Figure 5. Delays for VBR VoIP over WLAN

5.2 Extra Delay Incurred by the Multiplex-Multicast Scheme 
A VoIP packet will encounter extra delay at the MUX when it waits for the MUX to generate the next multiplexed packet. Recall that the MUX will send off one multiplexed packet to the AP once every $T$ seconds. Since we set the multiplexing period to be at most one audio-frame period in our study, our scheme ensures that the extra delay incurred at the MUX is bounded by one frame period ( $20 \mathrm{~ms}$ if GSM 6.10 codec is used). Note that only downlink packets go through the MUX.

To account for the extra delay, we define $M$ to be the random variable representing the extra multiplexing delay. We assume $M$ to be uniformly distributed between 0 and $20 \mathrm{~ms}$. Table 8 tabulates the distribution of multiplexing plus access delays incurred at the AP and the distribution of access delay incurred at the wireless stations. As shown, the local delay budget of $30 \mathrm{~ms}$ can be met comfortably for both CBR and VBR VoIP.

Table 7. Access Delay Distribution for Ordinary VoIP when System Capacity is Fully Used

\begin{tabular}{|c|c|c|c|c|}
\hline & \multicolumn{2}{|c|}{$\begin{array}{c}\text { Access delay for the } \\
\text { AP (Local delay for } \\
\text { downlink VoIP } \\
\text { packets) }\end{array}$} & \multicolumn{2}{c|}{$\begin{array}{c}\text { Access delay for the } \\
\text { station (Local delay } \\
\text { for uplink VoIP } \\
\text { packets) }\end{array}$} \\
\cline { 2 - 5 } & CBR(12) & VBR(25) & CBR(12) & VBR(25) \\
\hline $\operatorname{Pr}[\mathrm{A}<10 \mathrm{~ms}]$ & 1 & 0.900 & 0.999 & 1 \\
\hline $\operatorname{Pr}[\mathrm{A}<30 \mathrm{~ms}]$ & 1 & 0.990 & 1 & 1 \\
\hline $\operatorname{Pr}[\mathrm{A}<50 \mathrm{~ms}]$ & 1 & 1 & 1 & 1 \\
\hline
\end{tabular}

Table 8. Delay Distributions for Multiplex-Multicast Scheme when System Capacity is Fully Used

\begin{tabular}{|c|c|c|c|c|c|}
\hline $\begin{array}{c}\text { Access delay for the AP plus MUX } \\
\text { delay in the MUX (Local delay for } \\
\text { the downlink VoIP packets) }\end{array}$ & \multicolumn{3}{|c|}{$\begin{array}{c}\text { Access delay for the station } \\
\text { (Local delay for the uplink } \\
\text { VoIP packets) }\end{array}$} \\
\hline & $\begin{array}{c}\text { CBR } \\
(22)\end{array}$ & $\begin{array}{c}\text { VBR } \\
(46)\end{array}$ & & $\begin{array}{c}\text { CBR } \\
(22)\end{array}$ & $\begin{array}{c}\text { VBR } \\
(46)\end{array}$ \\
\hline $\operatorname{Pr}[\mathrm{M}+\mathrm{A}<10 \mathrm{~ms}]$ & 0.455 & 0.451 & $\operatorname{Pr}[\mathrm{A}<10 \mathrm{~ms}]$ & 0.996 & 0.992 \\
\hline $\operatorname{Pr}[\mathrm{M}+\mathrm{A}<20 \mathrm{~ms}]$ & 0.955 & 0.951 & $\operatorname{Pr}[\mathrm{A}<20 \mathrm{~ms}]$ & 1 & 0.999 \\
\hline $\operatorname{Pr}[\mathrm{M}+\mathrm{A}<30 \mathrm{~ms}]$ & 1 & 1 & $\operatorname{Pr}[\mathrm{A}<30 \mathrm{~ms}]$ & 1 & 1 \\
\hline
\end{tabular}

The delay results in this section show that the VoIP capacity we defined in the previous section using the loss metric can also meet the delay metric defined in this section. The Quality of Servive (QoS) of VoIP in terms of loss rate and delay is good enough for both ordinary VoIP and M-M VoIP.

\section{Conclusions}

This paper has proposed a Multiplex-Multicast (M-M) scheme for VoIP over WLAN. Our scheme aggregates downlink voice packets with header compression in the voice gateway, then multicasts the multiplexed packet to all the stations. The scheme can reduce the large overhead when VoIP traffic is delivered over WLAN. Unlike other VoIP capacity improvement schemes reported in the literature, the $\mathrm{M}-\mathrm{M}$ scheme requires no changes to the MAC protocol at the wireless end stations. Only when the VBR voice source is used, to reduce the loss rate for the downlink multicast packet, M-M scheme may need some minor modifications at the AP. This feature makes our scheme more readily deployable over the existing network infrastructure.

To test our proposed scheme, we set a performance target of i) no more than $1 \%$ VoIP packets can be lost; ii) no more $1 \%$ of the VoIP packets can experience more than $30 \mathrm{~ms}$ overall delay within the WLAN equipment and components introduced by our solutions. Our studies are comprehensive and include various voice codecs, CBR and VBR VoIP streams, and 802.11b, 802.11a, and 802.11g MAC protocols. The results show that our proposed scheme can achieve a voice capacity nearly $100 \%$ higher than ordinary VoIP in all cases, while meeting our performance target.

What we can conclude from the results of this paper is this. In an enterprise environment, the number of simultaneous VoIP sessions with the regular scheme will probably have to be reduced to around 5 for $802.11 \mathrm{~b}$ and $802.11 \mathrm{~g}$ to make room for traffic of other applications. This may result in an unacceptably high blocking probability for VoIP in many situations. Our Multiplex-Multicast scheme is one way to increase the VoIP capacity and decrease the blocking probability.

\section{References}

[1] Yi-Bing Lin and Imrich Chlamtac, Wireless and Mobile Network Architectures, John Wiley and Sons, 2001.

[2] Bill Douskalis, IP Telephony, the integration of robust VoIP services, Prentice Hall PTR, 2000.

[3] D. Chen, S. Garg, M. Kappes, and K. Trivedi, "Supporting VBR VoIP Traffic in IEEE 802.11 WLAN in PCF Mode," Technical Report ALR-2002-026, Avaya Labs, 2002.

[4] M. Veeraraghavan, N. Cocker and T. Moors, "Support of Voice Services in IEEE 802.11 Wireless LANs," INFOCOM 2001, Vol. 1, Apr. 2001, pp. 488-497.

[5] Rusty O. Bladwin, Nathaniel J. Davis IV, Scott F. Midkiff and Richard A. Raines, "Packetized Voice transmission using RT-MAC, a wireless real-time medium access control protocol", Mobile Computing and Communications Review, Vol 5, No.3, pp. 11-25, July, 2001.

[6] S. Garg and M. Kappes, "On the Throughput of $802.11 \mathrm{~b}$ Networks for VoIP," Technical Report ALR-2002-012, Avaya Labs, 2002. http://www.research.avayalabs.com/techreportY.html 
[7] S. Garg and M. Kappes, "An Experimental Study of Throughput for UDP and VoIP Traffic in IEEE 802.11b Networks," IEEE WCNC 2003, Vol. 3, March 2003, pp. 1748-1753.

[8] T. Hiraguri, T. Ichikawa, M. Iizuka, and M. Morikura, "Novel Multiple Access Protocol for Voice over IP in Wireless LAN," IEEE International Symposium on Computers and Communications, July 2002.

[9] A. Banchs, X. Perez, M. Radimirsch, H. Stuttgen, "Service Differentiation Extensions for Elastic and RealTime Traffic in 802.11 Wireless LAN," IEEE Workshop on High Performance Switching and Routing, May 2001, pp. 245-249.

[10] J. Kuri and S. K. Kasera, "Reliable Multicast in Multiaccess Wireless LANs," INFOCOM'99, Vol. 2, Mar. 1999, pp. 760-767.

[11] Min-Te Sun, Lifei Huang, Arora, A. and Ten-Hwang Lai, "Reliable MAC layer multicast in IEEE 802.11 wireless networks," Parallel Processing, Intl. Conf. on, Aug. 2002, pp. 527-536.

[12] Tang, K. and Gerla, M., "MAC Layer Broadcast Support in 802.11 Wireless Networks," MILCOM 2000, vol. 1, Oct. 2000, pp. 544-548.

[13] Sze, H.P., Liew, S.C., Lee, J.Y.B. and Yip, D.C.S, “A multiplexing scheme for H.323 voice-over-IP applications", IEEE J. Select. Areas Commun, Vol. 20, pp. 1360-1368, Sept. 2002.

[14] Hung-Huang Liu and Jean-Lien C. Wu, "Packet telephony support for the IEEE 802.11 wireless LAN", IEEE Communications Letters, Vol. 4, No. 9, pp. 286288, Sept. 2000.

[15] Matthew S. Gast, 802.11 Wireless Networks, the definitive guide, O'REILLY, 2002.

[16] Neeli Prasad and Anand Prasad, WLAN Systems and Wireless IP for Next Generation Communications, Artech House, 2002.

[17] A. Prasad, "Performance Comparison of Voice over IEEE 802.11 Scheme," IEEE VTC 1999, Vol. 5, Sept. 1999, pp. 2636-2640

[18] S. Casner and V. Jacobson, "Compressing IP/UDP/RTP headers for low-speed serial links", IETF RFC 2508, 1999.

[19] P. Brady, "A Model for Generating On-Off Speech Patterns in Two-Way Conversation," Bell Syst. Tech. Journal, vol. 48, no. 7, pp. 2245-2272, Sept. 1969.

[20] "The Network Simulator - ns2", http://www.isi.edu/nsnam/ns

[21] J. Feigin, K. Pahlavan, and M. Ylianttile, "HardwareFitted Modeling and Simulation of VoIP over a Wireless LAN," $52^{\text {nd }}$ IEEE Vehicular Technology Conference, Vol. 3, Sep 2000, pp. 1431-1438.

[22] T. J. Kostas, M. S. Borella, I. Sidhu, g. M. Schuster, J. Grabiec and J. Mahler, "Real-time voice over packet- switched networks,", IEEE Network, vol. 12, No. 1, pp. 18-27, January/February 1998.

[23] Philip K. McKinley and Suraj Gaurav, "Experimental evaluation of forward error correction on multicast audio streams in wireless LANs", Proceedings of the eighth ACM international conference on Multimedia, pp. 416418, 2000.

[24] B. Crow, I. Widjaja, J. Kim, P. Sakai, "IEEE 802.11 Wireless Local Area Networks," IEEE Communications Magazine, Vol. 35, Issue 9, Sept. 1997, pp. 116-126.

[25] D. Chen, S. Garg, M. Kappes, and K. Trivedi, "Supporting VoIP Traffic in IEEE 802.11 WLAN with Enhanced Medium Access Control (MAC) for Quality of Service," Technical Report ALR-2002-025, Avaya Labs, 2002. 